\title{
Aplicaciones actuales de la hipnosis clínica en Latinoamérica: una revisión sistemática de la literatura
}

\author{
Current Applications of Clinical Hypnosis in Latin America: A Systematic \\ Review of the Literature \\ Aplicações atuais da hipnose clínica na fmérica Latina: uma revisão \\ sistemática da literatura
}

\author{
Estefania Castañeda* \\ Universidad Eafit, Medellín, Colombia. \\ Alicia Krikorian** \\ Universidad Pontificia Bolivariana, Medellín, Colombia.
}

Doi: http://dx.doi.org/10.12804/revistas.urosario.edu.co/apl/a.5488

\section{Resumen}

La hipnosis es un tema controversial y estigmatizado. Las múltiples investigaciones de las últimas décadas han permitido tanto derrumbar mitos, como evidenciar sus mecanismos y aplicaciones, y cómo estos contribuyen al bienestar de las personas. El objetivo de este trabajo es describir las aplicaciones actuales de la hipnosis clínica en el contexto latinoamericano. Con este fin se realizó una revisión sistemática de la literatura en las bases de datos Lilacs y SciELo, empleando los términos "hipnosis", "hipnosis clínica" y "Latinoamérica". Se incluyeron revisiones de la literatura, artículos originales que tuvieran poblaciones latinoamericanas y presentaciones de caso, publicados entre 1990 y el 2016, escritos en español. La búsqueda inicial arrojó 202 artículos; después de eliminar replicaciones y los que no cumplían criterios de inclusión, 20 artículos fueron incluidos en la revisión. Las aplicaciones de la hipnosis descritas incluyen manejo del dolor, preparación a la cirugía, tabaquismo, problemas gastrointestinales y otras condiciones médicas, aplicaciones en niños y adolescentes, etcétera. Desafortunadamente, los estudios revisados tienen importantes falencias metodológicas, lo que dificulta extraer conclusiones sólidas. A pesar de que la hipnosis es eficaz, práctica, costo-efectiva y aplicada en una gran variedad de problemas clínicos, la evidencia que avala su uso en el

* Estefania Castañeda, Programa de Psicología, Universidad Eafit, Medellín, Colombia.

** Alicia Krikorian, Grupo de Dolor y Cuidado Paliativo, Escuela de Ciencias de la Salud, Universidad Pontificia Bolivariana, Medellín, Colombia.

La correspondencia relativa a este artículo debe ser enviada a Alicia Krikorian, Escuela de Ciencias de la Salud, Universidad Pontificia Bolivariana. Campus de Robledo Calle 78b No. 72a-109, Medellín, Colombia. Correo electrónico: aliciakriko@gmail.com

Cómo citar este artículo: Castañeda, E. \& Krikorian, A. (2018). Aplicaciones actuales de la hipnosis clínica en Latinoamérica: una revisión sistemática de la literatura. Avances en Psicología Latinoamericana, 36(2), 269-283. DOI: http://dx.doi. org/10.12804/revistas.urosario.edu.co/apl/a.5488 
contexto latinoamericano es muy limitada. Se requieren investigaciones con una metodología más rigurosa que permitan evidenciar su eficacia en este contexto. Palabras clave: hipnosis, usos terapéuticos, terapia, resultados del tratamiento, evaluación de resultados de intervenciones terapéuticas, América Latina, salud.

\section{fbstract}

Hypnosis is a controversial issue given the opposing positions it generates. Although saddled with a huge stigma, in recent decades research in the field has overthrew myths and demonstrated with empirical evidence its mechanisms and applications in many physical and psychological problems, contributing to the wellbeing and quality of patients. The aim of this paper is to describe the current applications of clinical hypnosis in the Latin American context. A systematic search of the literature was performed using the keywords 'hypnosis', 'clinical hypnosis' and 'Latin America' in Lilacs and SciELO databases. Literature reviews, original articles including Latin American population and case presentations, published between 1990 and 2016, and written in Spanish were included. A total of 202 articles were found, after removing replications and excluding articles that did not met the inclusion criteria only 20 articles were chosen. The applications of hypnosis described in the literature include pain management, preparation for surgery, smoking cessation, gastrointestinal problems and other medical conditions, applications in children and adolescents, among others. However, most of the results of the articles included have important methodological flaws, making it difficult to draw solid conclusions. Although hypnosis is cost-effective, practical, useful and can be applied for a variety of clinical problems, there is little evidence to support its use in our context. Research including Latin American samples and using more rigorous methodologies is needed to confirm its clinical validity in our context.

Keywords: hypnosis, therapeutic uses, therapy, treatment outcomes, evaluation of results of therapeutic interventions, Latin America, health.

\section{Resumo}

A hipnose é um tema controverso e estigmatizado. As múltiplas pesquisas das últimas décadas têm permitido derrubar mitos e evidenciar os seus mecanismos e aplicações, e como estes contribuem ao bem-estar das pessoas. O objetivo deste trabalho é descrever as aplicações atuais da hipnose clínica no contexto latino-americano. Para este fim se realizou uma revisão sistemática da literatura nas bases de dados Lilacs e SciELO empregando os termos "hipnose", "hipnose clínica" e "Latino-América". Se incluíram revisões da literatura, artigos originais que incluíram populações latino-americanas e apresentações de caso, publicados entre 1990 e 2016, escritos em espanhol. A busca inicial resultou em 202 artigos, depois de eliminar replicações e os que não cumpriam critérios de inclusão, 20 artigos foram incluídos na revisão. As aplicações da hipnose descritas incluem controle da dor, preparação à cirurgia, tabaquismo, problemas gastrointestinais e outras condições médicas, aplicações em crianças e adolescentes, entre outras. Desafortunadamente, os estudos revisados têm importantes falências metodológicas o que dificulta extrair conclusões sólidas. A pesar de que a hipnose é eficaz, prática, custo-efetiva e aplicada em uma grande variedade de problemas clínicos, a evidência que avaliza o seu uso no contexto latino-americano é muito limitada. Requerem-se pesquisas com uma metodologia mais rigorosa que permitam evidenciar a sua eficácia neste contexto.

Palavras-chave: hipnose, usos terapéuticos, terapia, resultados do tratamento, evaluación de resultados de intervenciones terapêuticas, América Latina, salud.

\section{Introducción}

La hipnosis ha sido un tema controversial debido a las posiciones antagónicas que genera: por una parte, carga con un importante estigma por el cual es generalmente rechazada y, por otra parte, en las últimas décadas se han realizado múltiples 
investigaciones que han permitido derrumbar los mitos que hay sobre ella, evidenciar sus mecanismos, las contribuciones que puede traer al bienestar y a la mejora de la calidad de vida de las personas, y demostrar que es una herramienta terapéutica de gran utilidad para una serie de problemas físicos y psicológicos (Jensen \& Patterson, 2014; Palsson, 2015; Tortosa, Gonzales \& Miguel, 1999; Vanhaudenhuysea, Laureysa \& Faymonville, 2014).

A pesar de la abundante literatura científica que soporta esta técnica (Alarcón \& Capafons, 2006; Capafons, 1999; Lynn \& Kirsch, 2005; Rodríguez \& Rodríguez, 2011; Tortosa et al., 1999), aún existe gran desconocimiento acerca del concepto de hipnosis clínica, sus aplicaciones y sus beneficios; ello se ve reflejado en la escasa información y educación al respecto, particularmente en las facultades de psicología y los programas de entrenamiento clínico. De hecho, muchos estudiantes y profesionales desconocen su historia y actualidad, lo que tiende a perpetuar las concepciones erróneas en torno a ella (Capafons, 2005).

La historia de la hipnosis es extensa y compleja, $y$, aunque hacer referencia a esta excede los propósitos del artículo, aproximarse a sus orígenes puede permitir, en parte, la comprensión de las problemáticas relacionadas en la actualidad con su definición y aplicaciones en el ámbito de la salud.

Existe evidencia del empleo de la hipnosis desde antiguas civilizaciones (Mac Hovec, 1975). Sin embargo, se suele mencionar a Mesmer como uno de los principales precursores de la hipnosis moderna y, en efecto, fue él quien introdujo el concepto de sugestionabilidad en el siglo XVIII de la mano de su teoría del "magnetismo animal" para explicar los fenómenos resultantes de su uso en el contexto clínico. Desafortunadamente, los resultados terapéuticos que obtuvo a través del uso de la sugestión fueron desacreditados por la Academia de Medicina de Francia y sus métodos condenados hasta el punto de obligarle a retirarse de la práctica médica. A pesar de ello, sus ideas continuaron teniendo una influencia en el mundo occidental (Gravitz, 1988; Tortosa et al., 1999).

Más tarde, Amand Marie Jacques de Chastenet empleó el término "sonambulismo magnético" para describir estados intermedios entre el sueño y la vigilia en los que se podían producir cambios, particularmente en la memoria y la identidad. José Custodio de Faria sustituyó los procedimientos de "pases magnéticos" por sugestiones hipnóticas, y solo hasta 1843 James Braid acuñó el término "hipnosis" y le dio mayor relevancia a los procesos de sugestión; con ello se sentaron las bases para entender que los efectos de la hipnosis se derivaban de la propia persona y no de una fuerza magnética, proponiendo así una explicación psicológica al fenómeno y dando inicio a su estudio científico (Pintar \& Lynn, 2009).

Hacia finales de 1800, Liebeault y Bernheim (de la Escuela de Nancy) fundaron la primera academia de hipnosis en Europa y comenzaron a examinar las reacciones fisiológicas derivadas de ella, considerándola un estado alterado de conciencia, y un método de tratamiento válido en medicina y neurología. En contraposición, la Escuela de la Salpêtrière, liderada por Charcot, sostenía que la hipnotizabilidad era un signo de patología mental (en la cual solo aquel que sufriera de neurosis de tipo histérico podía ser hipnotizado), mientras que los miembros de la Escuela de Nancy defendían que era un fenómeno que caía en el rango de lo normal y que las personas tenían diferentes grados de sugestionabilidad, pudiendo ser, por tanto, hipnotizables. Muchos clínicos y académicos (entre ellos Freud) acogieron de forma temporal la hipnosis, sin embargo, no estuvieron libres de rechazos, críticas y replanteamientos. Ello llevó a que su uso cayera en detrimento (Gravits, 1988; Pintar \& Lynn, 2009; Tortosa et al., 1999).

No obstante, en los años sesenta hay un resurgir del estudio de la hipnosis, partiendo de una mirada más científica y buscando comprender los procesos implicados en la sugestión y sus efectos (British 
Society of Psychology, 2001). Se retoman así los usos terapéuticos de la hipnosis y se comienza a estudiar su efectividad en una variedad de problemas clínicos. Incluso, se proponen diversas aproximaciones como la hipnosis despierta y la autohipnosis (Capafons, 1999, 2004; Martínez-Tendero, Capafons, Weber \& Cardeña, 2001).

La hipnosis es actualmente definida por la Asociación Americana de Psicólogos (APA, 2014) como "un estado de conciencia que implica la atención focalizada y conciencia periférica reducida, caracterizado por una mayor capacidad de respuesta a la sugestión". Por su parte, la Sociedad Británica de Psicología (2001) indica que la hipnosis denota una interacción entre dos personas, en la cual una de ellas ("el hipnotizador") busca influir sobre las percepciones, los sentimientos, los pensamientos y las conductas de la otra pidiéndole que se concentre en imágenes o ideas que evocan el efecto buscado. Las indicaciones verbales empleadas se denominan "sugestiones", tienen una cualidad de involuntariedad y pueden ser de tipo ideomotor, de experiencias perceptivas, de experiencias complejas o poshipnóticas. Las personas pueden incluso aprender a aplicar los procedimientos por sí mismos, logrando la autohipnosis. De otro lado, la Asociación para el Avance de la Hipnosis Experimental y Aplicada (2017) define la hipnosis como una construcción social que denota un rango de interacciones entre personas (el hipnotizado y el hipnotizador) en las que se activan roles a partir de la influencia social. En este proceso de acuerdo implícito, "el hipnotizador/es intenta/n influir en los sentimientos, el pensamiento y la conducta, de las otra/s persona o personas pidiéndole/s que se concentre/n en ideas e imágenes que pueden evocar los efectos deseados".

El estado hipnótico implica un estado en el cual los individuos reportan un cambio en el estado de conciencia con una mayor capacidad de absorción y disociación, que se diferencia del estado de vigilia y que no implica una pérdida del control personal. Es requisito indispensable para el logro del estado hipnótico que haya una cooperación por parte de quien es hipnotizado. De hecho, la sugestión juega un papel muy importante dentro de este proceso, pues posibilita que la persona llegue a ser hipnotizada y pueda seguir las sugerencias que se le hacen. Adicionalmente, se dan cambios a nivel de la conciencia que incluyen el reposo mental, el estar absorto, la reducción de la autoorientación y la automaticidad (Vanhaudenhuysea et al., 2014). Incluso, se han evidenciado procesos de tipo neurofisiológico frente a estos cambios, a la vez que se han comenzado a describir algunos mecanismos cerebrales implicados (Jensen \& Patterson, 2014; Lanfranco, Canales-Johnson \& Huepe, 2014).

La hipnosis clínica es aplicada en diferentes problemáticas, como el dolor (agudo y crónico), la ansiedad, el tabaquismo, los trastornos de estrés postraumático, los trastornos gastrointestinales y otros problemas de salud (Jensen \& Patterson, 2014; Landolt \& Milling, 2011; Lanfranco et al., 2014; Lynn \& Kirsch, 2005; Mendoza \& Capafons, 2009; Palsson, 2015). Gracias a la evidencia empírica y a una cuidadosa observación clínica, se ha logrado debatir una serie de mitos, llegando a un consenso científico sobre algunas características de la hipnosis, entre las que se encuentran las siguientes: la hipnosis no es un estado de sueño; bajo hipnosis las personas tienen la capacidad de controlar sus conductas y son conscientes de su entorno; no implica ningún peligro si es realizada por profesionales cualificados; la amnesia poshipnótica es poco frecuente y usualmente reversible cuando ocurre; la hipnosis podría provocar falsos recuerdos, tal como ocurre en el estado de vigilia (Capafons, 1998).

La utilidad y eficacia de la hipnosis han sido evidenciadas en múltiples contextos (Jensen \& Patterson, 2014; Landolt \& Milling, 2011; Lanfranco et al., 2014; Mendoza \& Capafons, 2009; Palsson, 2015), aunque algunos autores indican la necesidad de incrementar el rigor metodológico para lograr resultados más robustos en contextos 
específicos (Molina, Mendoza, Capafons \& Llanes, 2016; Molina, Mendoza, Díez, Llanes \& Capafons, 2016). Puntualmente, se desconoce el estado actual de la investigación en torno a sus aplicaciones clínicas en Latinoamérica. Por tanto, el objetivo del presente estudio fue realizar una revisión exhaustiva y sistemática de la literatura científica con el fin de identificar las actuales aplicaciones de la hipnosis clínica en el contexto latinoamericano. Se pretende con ello aportar a una mayor claridad en torno a su conceptualización, sus aplicaciones clínicas y su utilidad, y se espera asimismo que tenga un efecto desmitificador de esta práctica en el ámbito clínico y psicológico.

\section{Metodología}

Se llevó a cabo una revisión sistemática de la literatura siguiendo las recomendaciones de Beltrán (2005) y la guía para revisiones sistemáticas Prisma (Urrutia, 2010): (1) se estableció como pregunta de investigación: ¿cuáles son las aplicaciones actuales de la hipnosis clínica en Latinoamérica?; (2) se especificaron los siguientes criterios de inclusión: artículos originales que incluyeran población latinoamericana, revisiones de la literatura y presentaciones de caso, publicados a partir de 1990 y hasta diciembre del 2016, escritos en español; como criterios de exclusión: editoriales y comentarios, estudios originales publicados antes de 1989, que no incluyeran poblaciones latinoamericanas y que no fueran escritos en idioma español; (3) para el plan de búsqueda de la literatura, se seleccionaron las bases de datos SciELO y Lilacs; (4) se establecieron como palabras clave "hipnosis", "hipnosis clínica" y "Latinoamérica"; (5) posteriormente, se realizó un registro de los artículos encontrados en una tabla de Excel con información de cada uno; (6) finalmente, se interpretaron y analizaron los resultados según las aplicaciones clínicas más frecuentemente encontradas. Los resultados son presentados a continuación.

\section{Resultados}

Los resultados de búsqueda en la base de datos SciELO arrojaron 47 artículos y en Lilacs 184. De forma adicional, se incluyeron 2 artículos a través de búsqueda manual. Después de eliminar las replicaciones, se obtuvo un total de 202 artículos. De estos, solo 20 cumplieron los criterios de inclusión (se eliminaron artículos que estaban escritos en un idioma diferente al español, que no abordaban específicamente la aplicación de hipnosis clínica, estudios originales que no incluían población latinoamericana, o en los que no fue posible encontrar los textos completos). Se encontraron 10 artículos de revisión, 3 estudios de caso, 2 estudios descriptivos, transversales y retrospectivos, 2 estudios comparativos, 2 ensayos fase II, y un estudio cualitativo descriptivo (gráfico 1).

A continuación se detallan los resultados según las aplicaciones clínicas de la hipnosis reportadas y que son sintetizadas en la tabla 1.

\section{Manejo del dolor}

Quizás uno de los usos más frecuentes de la hipnosis en la literatura es en el control del dolor. En la presente revisión se encontraron cuatro artículos, dos estudios originales y dos revisiones. Pinto y Montoya (2010) realizaron un estudio de serie de cinco casos de pacientes quemados en los que se empleó la hipnosis durante el proceso de curación de heridas. Reportan la disminución en las puntuaciones de dolor (según la Escala Visual Análoga) antes y después de la intervención. Desafortunadamente, el estudio cuenta con graves falencias metodológicas y, a pesar de que hubo una aparente eficacia de la técnica, no es posible extraer conclusiones sólidas. Otro estudio, llevado a cabo en Cuba, comparó la eficacia de la hipnosis para el tratamiento de la cefalea vs. el tratamiento médico convencional (Cos, Semanat \& Moya, 2000). Participaron en total 756 personas 


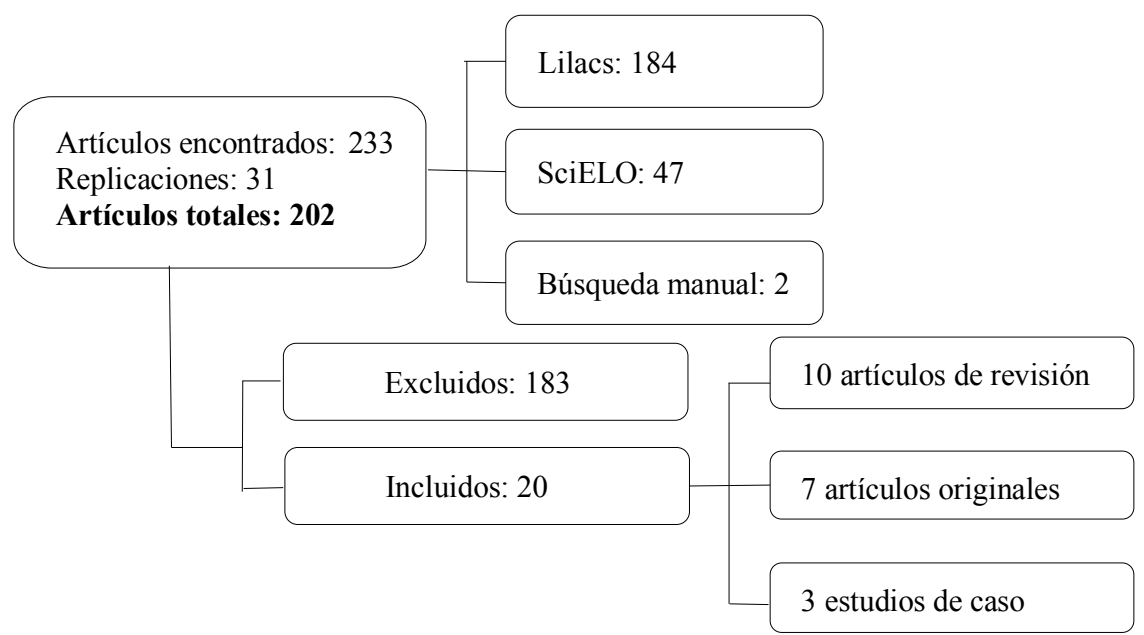

Gráfico 1. Resultados de la búsqueda de la literatura

Tabla 1

Resumen de los artículos incluidos en la revisión sistemática

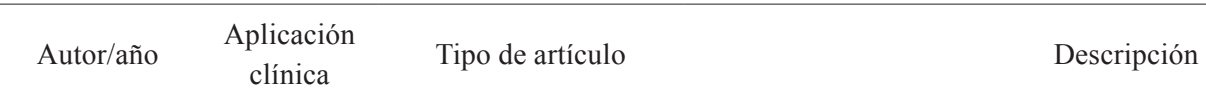

Se realizó una revisión de la literatura sobre guías de práctica clínica con el fin de generar recomendaciones de práctica clínica sobre eficacia y seguridad del tratamiento para la cesación de la adicción al tabaco en adultos colombianos. Dentro de los tratamientos resul-

Alba et al., Consumo 2013 de tabaco
Revisión (sistemática)

tantes estuvo la hipnosis. Los estudios encontrados para hipnosis fueron escasos y de calidad deficiente, por lo que el consenso de expertos consideró que no existe evidencia suficiente que respalde el uso de la hipnosis como tratamiento para la cesación de la adicción al tabaco. No incluye estudios realizados con población latinoamericana.

Se hace una revisión de las estrategias psicoterapéuticas para el manejo de la eyaculación precoz, incluida la hipnosis. Se describen

Uribe- Eyaculación

Holguin, 2010 precoz

Revisión (sistemática) de manera general los objetivos y las técnicas hipnóticas empleadas en su tratamiento. No incluye estudios realizados con población latinoamericana.

$\begin{array}{lll}\text { Samano \& } & \text { Control del } & \text { Revisión (narrativa) } \\ \text { Abia, } 1996 & \text { dolor }\end{array} \quad$

Abia, 1996

Se hace una revisión de las aplicaciones de la hipnosis ericksoniana en anestesia y se reporta de forma general la experiencia de su aplicación en una institución hospitalaria.

Se hace una revisión sistemática de la literatura disponible acerca de la hipnosis como técnica para dejar de fumar. La calidad de los

Ayala, 2010

Consumo de tabaco
Revisión (sistemática) ios disponibles es limitada. Se concluye que es necesario rearvenciones multidimensionales combinando diversas estrategias terapéuticas que incluyan la hipnosis. No incluye estudios realizados con población latinoamericana. 


\begin{tabular}{clll}
\hline Autor/año & $\begin{array}{c}\text { Aplicación } \\
\text { clínica }\end{array}$ & Tipo de artículo & \multicolumn{1}{c}{ Descripción } \\
\hline \multirow{2}{*}{ Bragado, 2009 } & $\begin{array}{l}\text { Pediatría } \\
\text { oncológica }\end{array}$ & Revisión (narrativa) & $\begin{array}{l}\text { Describe intervenciones psicooncológicas en pediatría de modo } \\
\text { general e incluye un apartado en el que se describe la aplicación de } \\
\text { la hipnosis principalmente para su uso en procedimientos médicos } \\
\text { dolorosos. No incluye estudios realizados con población latinoame- } \\
\text { ricana. }\end{array}$ \\
\hline
\end{tabular}

$\begin{array}{ll}\text { Castillo et al., } & \begin{array}{l}\text { Hipnoinmu- } \\ \text { noterapia }\end{array} \\ \text { Presentación de caso }\end{array}$

Claros, 2008 $\begin{aligned} & \text { Hipomoti- } \\ & \text { lidad coló- } \\ & \text { nica }\end{aligned} \quad$ Presentación de caso

Cos, Semanat
$\&$ Moya, 2000 Cefalea Artículo original
Se presenta el caso de una adolescente con alopecia crónica asociada a inmunodeficiencia celular. Se describe el uso de hipnosis como coadyuvante del tratamiento con inmunoterapia. Las lesiones desaparecieron y los resultados positivos del tratamiento se mantuvieron a un año.

Se describe la aplicación de la hipnosis en un caso de hipomotilidad colónica. Se detalla la técnica empleada y los resultados del tratamiento con hipnosis de forma cualitativa.

Se describe un estudio de un ensayo clínico aleatorizado cuyo propósito fue evaluar la eficacia de la sugestión para el tratamiento de la cefalea y calcular su valor económico, en contraste con el tratamiento farmacológico. Se hicieron evaluaciones de los efectos del tratamiento a los 10 y 60 minutos. Se concluye que la sugestión hipnótica es útil para aliviar la cefalea y es más costo-efectiva.

Se reporta un estudio descriptivo, transversal y retrospectivo, acerca del uso de la hipnosis analgésica como coadyuvante en cirugía ambulatoria en pacientes oncológicos en una población cubana. Los autores concluyen que la cirugía, acompañada de analgesia hipnótica o anestesia acupuntural, presenta resultados satisfactorios y se disminuye el coste hospitalario.
Infante \& Rodríguez, Cirugía Artículo original 2006

Lanfranco, 2010 Depresión
unipolar Revisión (narrativa)
Aborda los aspectos históricos de la hipnosis, su definición, sus correlatos neurobiológicos y sus aplicaciones en psicología clínica. Incluye un apartado en el que se describe específicamente la utilidad de la hipnosis para el tratamiento de la depresión unipolar. No incluye estudios realizados con población latinoamericana.

El artículo revisa el estado de la evidencia empírica de la eficacia de la hipnosis en diversos problemas clínicos. Los autores concluyen que su eficacia está bien establecida en aplicaciones como el manejo del dolor y otras condiciones médicas, hay evidencia aceptable

Mendoza \& AplicacioCapafons, nes clínicas Revisión (narrativa) 2009 diversas de su eficacia en el tratamiento de la depresión, los trastornos del sueño, dejar de fumar, la obesidad, el asma y la enuresis infantil. Así mismo, cuando se utiliza la hipnosis como un coadyuvante a otras intervenciones médico-psicológicas, incrementa la eficacia o eficiencia de tales intervenciones. No incluye estudios realizados con población latinoamericana.

Se describen los resultados de un estudio descriptivo y transversal en el que se examinaron los efectos de la hipnosis preoperatoria orientada a la disminución del miedo quirúrgico. Los resultados mostraron una eficacia satisfactoria de la implementación de la hipnosis como un complemento al ayudar a eliminar tensiones, temor, ansiedad y a disminuir el estrés.
Muñiz et al., Cirugía Artículo original
2013 


\begin{tabular}{|c|c|c|c|}
\hline Autor/año & $\begin{array}{l}\text { Aplicación } \\
\text { clínica }\end{array}$ & Tipo de artículo & Descripción \\
\hline $\begin{array}{l}\text { Oliveros et al., } \\
2015\end{array}$ & $\begin{array}{l}\text { Gingivitis } \\
\text { descamativa } \\
\text { crónica }\end{array}$ & Presentación de caso & $\begin{array}{l}\text { Se describe la aplicación de la hipnosis en combinación con otras } \\
\text { terapias (neural, acupuntura) en un caso de gingivitis descamativa. }\end{array}$ \\
\hline $\begin{array}{l}\text { Pérez et al., } \\
2008\end{array}$ & $\begin{array}{l}\text { Enuresis } \\
\text { nocturna }\end{array}$ & Artículo original & $\begin{array}{l}\text { Se reportan resultados de un ensayo clínico no aleatorizado com- } \\
\text { parando la hipnosis con el uso de imipramina para el control de } \\
\text { la enuresis nocturna en niños y adolescentes }(\mathrm{n}=20) \text {. Los autores } \\
\text { concluyen que el tratamiento basado en la hipnosis fue tan eficaz } \\
\text { como el farmacológico, con la ventaja de que no hubo efectos se- } \\
\text { cundarios. }\end{array}$ \\
\hline
\end{tabular}

Estudio descriptivo, de casos sucesivos, con cinco participantes con

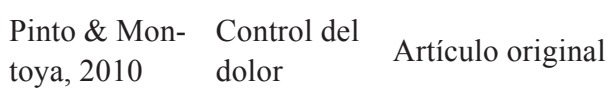
quemaduras de segundo grado profundo. Se examinó la utilidad de la hipnosis y la autohipnosis para el control del dolor durante las curaciones, a través de una metodología mixta cuanti-cualitativa. Los autores concluyen que la hipnosis fue eficaz en la reducción del dolor en estos pacientes.

$\begin{array}{ll}\text { Quiroz \& } & \begin{array}{l}\text { Manejo de } \\ \text { conducta en } \\ \text { niños }\end{array}\end{array}$

Rodríguez \& Rodríguez, 2011 Funciones cognitivas

Artículo original

Tobón et al., Dispepsia 2003 funcional

Revisión (narrativa)

Se hace una revisión de la literatura sobre técnicas no convencionales para el manejo de las conductas en niños (hipnosis, musicoterapia, distracción audiovisual y aromaterapia). Aunque los autores indican que es sistemática, no reportan la metodología de búsqueda.

Se realizó un estudio analítico experimental en el que se examinó el desempeño de la memoria en vigilia y bajo hipnosis en 19 estudiantes elegidos al azar. Los resultados indicaron mejor desempeño en la memorización y la evocación bajo hipnosis muy profunda que en la memorización en vigilia recordada.

Se hace una revisión crítica sobre el tratamiento psicológico de la dispepsia funcional. Se incluye la utilidad y la eficacia de la hipnoterapia. Se analizan los efectos de diversas terapias psicológicas y se concluye que todas tienen efectos significativos sobre la reducción de los síntomas dispépticos. Con respecto a la hipnosis, esta ha demostrado efectos significativos sobre algunas variables fisiológicas relacionadas con la dispepsia. No incluye estudios realizados con población latinoamericana.

Se describe el abordaje psicológico del dolor crónico y las diferentes aproximaciones clínicas disponible. Se menciona la utilidad de la hipnosis, pero no se profundiza en ello. No incluye estudios realizados con población latinoamericana.

Se describen los resultados de un ensayo clínico aleatorizado con grupo control comparando hipnosis y tratamiento convencional.

Zayas et al., Hiperten$2003 \quad$ sión arterial Artículo original Los autores reportan diferencias que indican un mayor control sintomático en el grupo experimental, pero las pruebas estadísticas reportadas son insuficientes para concluir sobre ello (no se aportan niveles de significación estadística). 
que fueron asignadas de forma aleatoria al grupo experimental o control. El efecto de la hipnosis fue evaluado de manera categórica (sentirse mejor, igual o peor) inmediatamente después de usar la técnica y una hora después. Así mismo, se comparó el costo económico del tratamiento en los dos grupos. Los autores concluyen que la sugestión hipnótica es efectiva para aliviar este tipo de dolor $\mathrm{y}$, al mismo tiempo, se obtiene un ahorro por la disminución en el uso de fármacos. Nuevamente, el estudio mencionado cuenta con limitaciones metodológicas que restan validez a los hallazgos. Respecto a los artículos de revisión, Vallejo (2008) describe las diversas estrategias terapéuticas no farmacológicas disponibles para el manejo del dolor. Puntualmente especifica que la hipnosis es útil cuando se busca que los sistemas de regulación natural del dolor operen adecuadamente, pero no profundiza en la evidencia empírica que soporta esta afirmación. Por su parte, Mendoza y Capafons (2009) afirman, en su revisión acerca de la utilidad de la hipnosis para diferentes problemáticas en salud, que la hipnosis es un tratamiento eficaz, bien establecido y empíricamente validado para el manejo del dolor experimental, agudo o crónico. Basan sus conclusiones en una serie de estudios y revisiones sistemáticas, ninguno de ellos de origen latinoamericano.

\section{Cirugía}

Se encontraron cuatro artículos que abordan el uso de la hipnosis en procedimientos quirúrgicos. El primer estudio, de tipo descriptivo, transversal y retrospectivo, describe de forma general los resultados de la cirugía ambulatoria en pacientes oncológicos en una población cubana (Infante \& Rodríguez, 2006). La hipnosis de tipo analgésico fue empleada de forma complementaria en 62 de los 573 pacientes incluidos en el estudio. Si bien los autores concluyen que la cirugía acompañada de analgesia hipnótica o anestesia acupuntural presenta resultados satisfactorios y se disminuye el coste hospitalario, no se describe cómo examinaron dicho impacto ni los resultados comparativos en las cirugías en las que no emplearon la hipnosis. Desafortunadamente, la metodología empleada es de calidad deficiente, lo que imposibilita la extracción de conclusiones sobre la eficacia de la hipnosis analgésica como adyuvante. En un segundo estudio, Muñiz, Soto, López, Orellana y Vinent (2013) examinaron los efectos de la hipnosis preoperatoria orientada a la disminución del miedo quirúrgico en un grupo de 20 pacientes a los que se les realizó cirugía oral. Según los autores, los resultados mostraron una eficacia satisfactoria de la implementación de la hipnosis como un complemento al ayudar a eliminar tensiones, temor, ansiedad y a disminuir el estrés; también ayudó a evitar el aumento de la presión arterial durante el procedimiento, disminuyendo complicaciones transoperatorias o posoperatorias. Sin embargo, no se empleó grupo control, lo que hizo que no fuera posible comparar la eficacia con respecto a otras estrategias (Muñiz et al., 2013). El tercer artículo encontrado fue una revisión narrativa de la literatura que describe las generalidades de la hipnosis eriksoniana analgésica y su aplicación como coadyuvante en la preparación de la cirugía (Samano \& Abia, 2006). Finalmente, Mendoza y Capafons (2009) presentan en uno de los apartados de su revisión narrativa el uso de la hipnosis clínica para la preparación a la cirugía. Explican que se ha empleado como coadyuvante para aliviar la ansiedad relacionada con estos procedimientos, como técnica coadyuvante a la analgesia farmacológica y para enseñar estrategias de afrontamiento ante la cirugía. Así, ha permitido reducir la cantidad de medicación pre y poscirugía para el dolor, el sangrado y el tiempo de hospitalización, $\mathrm{y}$ ha facilitado el periodo posoperatorio y la recuperación. Según los resultados de los estudios referenciados en su revisión (ninguno de origen latinoamericano), concluyen que la hipnosis coadyuvante es útil para 
reducir el dolor y el malestar psicológico asociados a la cirugía, y que la evidencia existente indica que la hipnosis coadyuvante es superior al tratamiento médico estándar en términos de calidad y coste.

\section{Niños y adolescentes}

Se encontraron cuatro artículos (dos estudios originales y dos revisiones) que abordaban el uso de la hipnosis en niños y adolescentes. Pérez, Sosa, García, Pinto y Arias (2008) realizaron un ensayo clínico no aleatorizado comparando la hipnosis con el uso de imipramina para el control de la enuresis nocturna primaria no complicada y secundaria no complicada. Siguieron a 40 pacientes de Santiago de Cuba, con edades entre 7 y 18 años, durante un periodo de 3 meses. Evaluaron la funcionalidad familiar, el impacto de los tratamientos (usando 4 categorías: curado, mejor, igual o peor) y la respuesta terapéutica a los 90 días (satisfactoria o no satisfactoria). Sus resultados indicaron que $18 \mathrm{de}$ los 20 sujetos tratados tanto con hipnosis como con imipramina habían mejorado a los 45 días, efecto que se mantuvo hasta los 90 días. Según los autores, la hipnosis fue tan eficaz como el fármaco, ya que generó mejoría de los síntomas sin causar efectos adversos (Pérez et al., 2008). Otro artículo reportó un estudio de caso en el que se empleó hipnoinmunoterapia en una adolescente que presentaba alopecia desde el divorcio de los padres a sus 3 años de edad (Castillo, Toirac, Castillo \& Columbié, 2010). La paciente fue diagnosticada con "inmunodeficiencia celular e hipergammaglobulinemia por estrés". Se inició tratamiento con inmunoestimulantes celulares e hipnosis. Las lesiones por alopecia desaparecieron a los 6 meses y el cambio se mantuvo por un año. Desafortunadamente, no es posible determinar, según la información aportada, si el efecto terapéutico fue derivado del uso de hipnosis o de los otros tratamientos empleados. De los dos artículos de revisión uno aborda, entre otros aspectos, el uso de la hipnosis en niños con cáncer (Bragado, 2009), mientras que el otro revisa el uso de técnicas no convencionales, entre ellas la hipnosis, para problemas de conducta en niños (Quiroz \& Melgar, 2012). En el primero se afirma que la hipnosis es una de las intervenciones psicológicas que muestra un mayor efecto sobre las medidas de dolor y malestar asociadas a los procedimientos médicos, al igual que la distracción y la terapia cognitivo-conductual, y es de eficacia comprobada en la reducción de las náuseas/ vómito. En el segundo artículo, se explora el uso de la hipnosis en odontopediatría, reportando que es útil para problemas como la ansiedad dental, la fobia dental, el control del dolor, la tolerancia a los aparatos de ortodoncia, como complemento a la sedación por inhalación y la modificación de hábitos orales no deseados (chuparse el dedo, bruxismo, náuseas, etc.). Se describen asimismo las ventajas de la hipnosis sobre otras estrategias y se concluye que es una de las más satisfactorias, después de la distracción audiovisual. Cabe aclarar que ninguna de las dos revisiones mencionadas es sistemática y específica a la hipnosis $\mathrm{y}$, de nuevo, ninguno de los estudios incluidos en ellas emplea población latina.

\section{Tabaquismo}

Sobre el uso de la hipnoterapia para la cesación del consumo de tabaco se encontraron tres artículos de revisión de la literatura (Alba et al., 2013; Ayala, 2010; Mendoza \& Capafons, 2009). Mendoza y Capafons (2009) en su revisión encontraron que la evidencia respecto a su utilidad es controvertida, pero que se considera una estrategia igualmente eficaz que otros tratamientos y que cuenta con la ventaja de ser eficiente, breve y económica. En la revisión sistemática realizada por Ayala (2010), se incluyeron 51 estudios, y concluye que, si bien la calidad de los estudios es limitada, se requiere una estrategia de tratamiento multidimensional que incluya la suplencia de nicotina, técnicas comportamentales e hipnóticas, y estrategias para mantener la abstinencia a largo plazo. En particular, reporta 
cinco estudios que emplearon la hipnosis, ninguno de los cuales se llevó a cabo en poblaciones latinas. En ellos, la hipnosis fue usada como técnica aislada y como adyuvante, mejorando la adhesión terapéutica e incrementando la probabilidad de éxito terapéutico. Por su parte, Alba et al. (2013) realizaron una revisión sistemática de guías de práctica clínica empleando una adaptación de la metodología ADAPTE. De las estrategias reportadas, encontraron que la evidencia recogida en cinco fuentes (tres guías clínicas, una revisión sistemática y un ensayo clínico aleatorizado) no muestra beneficios de la hipnosis para mejorar las tasas de cesación a largo plazo y concluyen que no existe suficiente respaldo para el uso de la hipnosis como tratamiento para la cesación de la adicción al tabaco. Nuevamente, las fuentes provienen de poblaciones no latinoamericanas.

En síntesis, aunque no es posible negar una cierta efectividad de la hipnosis para la cesación del consumo de tabaco, no hay suficiente evidencia empírica y se requiere de más investigación sobre el tema, particularmente empleando poblaciones latinoamericanas.

\section{Problemas gastrointestinales}

Se encontró un artículo de revisión sobre el uso de hipnosis en dispepsia funcional, la cual es definida como el conjunto de síntomas abdominales del tracto gastrointestinal superior, no explicados por alteraciones orgánicas demostrables por los medios diagnósticos convencionales (Talley et al., 2000). En dicho artículo se realizó un análisis crítico de la literatura que comparó la eficacia de la hipnosis clínica y otras estrategias psicoterapéuticas (Tobón, Vinaccia \& Sandín, 2003). Según los autores, se concluyó que el uso de la hipnoterapia es efectiva en la reducción de síntomas dispépticos y psicopatológicos, además de reducir el número de visitas médicas y el consumo de fármacos. Es de aclarar que, si bien la revisión es realizada por investigadores latinoamericanos, los cuatro estudios sobre hipnoterapia en los que basan sus hallazgos fueron de poblaciones no latinas. En un segundo artículo se presenta el caso de una paciente de Bolivia con hipomotilidad colónica (Claros, 2008). La autora hace una descripción detallada de la técnica empleada y concluye que la hipnosis fue de utilidad, ya que generó alivio y sensación de bienestar. Sin embargo, no se reporta una línea base ni cómo ni durante cuánto tiempo se realizaron los seguimientos.

\section{Otros problemas médicos}

Se encontraron tres artículos que abordan el uso de la hipnosis en otras problemáticas de salud: gingivitis descamativa crónica, hipertensión arterial esencial y obesidad mórbida. Respecto a la primera condición, se encontró un estudio de caso de un paciente cubano en el cual el tratamiento convencional no generó mejoría y en el que decidieron usar hipnosis en combinación con terapia neural, acupuntural y periodontal. Como resultados, encontraron una disminución en el proceso inflamatorio e incremento de la autoestima y calidad de vida, sin embargo, no fue posible determinar los efectos individuales de la hipnosis dada la combinación de terapias (Oliveros, Ortiz, Pérez, Castellanos \& Cobián, 2015). En el caso de la hipertensión arterial, los autores realizaron un ensayo clínico aleatorizado que incluyó 87 personas en el grupo de estudio (donde se aplicó tratamiento hipnótico complementario) y 174 controles (Zayas, Romero, Alfonso \& Abreu, 2003). En ambos grupos examinaron el grado de eficacia del tratamiento (en función del grado de control de la hipertensión y a la disminución en uso de fármacos) y la satisfacción de los pacientes. En el grupo de estudio se logró un adecuado control de la tensión arterial en el $66,6 \%$ de los pacientes vs. $31,6 \%$ en el grupo control; la respuesta al tratamiento fue directamente proporcional al grado de susceptibilidad hipnótica y hubo una disminución clínica en el uso de fármacos (desafortunadamente, no se 
reportan niveles de significación estadística de las comparaciones). Finalmente, Mendoza y Capafons (2009) en su revisión mencionan la utilidad de la hipnosis como un coadyuvante "probablemente eficaz" en la terapia cognitivo-conductual de la obesidad mórbida.

\section{Funciones cognitivas}

Se halló un estudio que examinó los efectos de la hipnosis sobre la memoria en un grupo de estudiantes de enfermería de Cuba (Rodríguez \& Rodríguez, 2011). Se realizó un estudio analítico experimental en el que se examinó, a través del test de 10 palabras, el desempeño de la memoria en vigilia y bajo hipnosis en 19 estudiantes elegidos al azar. Crearon un modelo de hipnosis muy profunda, grupal y simultánea, con el fin de estudiar la memoria controlando los fenómenos hipnóticos por etapas. Utilizaron el test de las 10 palabras y aplicaron el paradigma fisiológico para diagnosticar el estado de conciencia y la definir la etapa "muy profunda". Los resultados indicaron mejor desempeño en la memorización y la evocación bajo hipnosis muy profunda que en la memorización en vigilia recordada.

\section{Depresión}

Se encontraron dos artículos de revisión narrativa (Lanfranco, 2010; Mendoza \& Capafons, 2009) en los cuales se revisa el uso de la hipnosis como adyuvante en el tratamiento de la depresión. En el primero se describe un estudio en el que se examinó la eficacia de la hipnosis como adyuvante en el tratamiento cognitivo-conductual de la depresión, encontrando que hubo un mayor impacto en depresión, ansiedad y desesperanza al usar la hipnosis que al no hacerlo. El estudio, sin embargo, no se realizó en el contexto latinoamericano (Mendoza \& Capafons, 2009). En el segundo artículo se detalla el modelo de Yapko, que implementa la hipnosis en combinación con la terapia cognitivo-conductual y la terapia estratégica. De igual manera que en las revisiones antes mencionadas, en el artículo se citan estudios en los que no se incluyen poblaciones latinoamericanas (Lanfranco, 2010).

\section{Discusión y conclusiones}

Según los resultados de la presente revisión sistemática, las aplicaciones clínicas más frecuentemente reportadas en el contexto latinoamericano coinciden con aquellas que son descritas a nivel mundial: manejo del dolor y de procedimientos médicos, tabaquismo, problemas gastrointestinales y otras condiciones médicas, y su uso con niños y adolescentes (Häuser, Hagl, Schmierer \& Hansen, 2016; Mendoza \& Capafons, 2009).

Se observa que la gran mayoría de los artículos de estudio revisados no obtiene resultados suficientemente sólidos para afirmar que la implementación de la hipnosis es eficaz en nuestro contexto: las metodologías de las investigaciones no son suficientemente rigurosas o no se reporta en los artículos los detalles de esta, y no son realizados en población latinoamericana. Desafortunadamente, ninguno de los artículos de revisión incluía estudios originales realizados con población latinoamericana. Los estudios de caso y los dos ensayos fase II encontrados, aunque sí incluyen población de Latinoamérica, presentan falencias en su metodología, lo que se traduce en una baja validez de los resultados. Sin embargo, es importante mencionar que la búsqueda de artículos se realizó a través de solo dos bases de datos, ambas de acceso libre, lo cual puede implicar una limitación del estudio. Probablemente en otras bases de datos de acceso restringido se puedan encontrar artículos de calidad superior y futuras revisiones deberían ampliar la estrategia de búsqueda.

Llama la atención que, a pesar de que la hipnosis es una estrategia terapéutica eficaz y poderosa para el tratamiento complementario de una serie de problemas médicos y psicológicos, además de 
ser costo-efectiva (lo cual la hace especialmente interesante en una región donde las limitaciones económicas abundan y las medidas terapéuticas útiles, prácticas y de fácil aplicación son muy necesarias), su uso en el contexto latinoamericano es bastante escaso y los estudios al respecto muy limitados en cantidad y calidad.

La revisión aquí presentada permite concluir que existe muy poca investigación sobre la hipnosis y su eficacia en población latinoamericana, y la gran mayoría de los estudios encontrados muestra bajo rigor metodológico, lo que hace que sea limitada la evidencia actual en nuestro contexto. Se requerirán futuras investigaciones que usen metodologías más apropiadas, en las cuales se expliquen en detalle las estrategias hipnóticas utilizadas, y se empleen diseños comparativos con evaluaciones pre y postratamiento, utilizando instrumentos validados que tomen muestras de tamaño apropiado y participantes latinoamericanos. Ello permitirá evidenciar la utilidad y la eficacia de esta técnica en nuestro medio, asunto relevante si se tiene en cuenta su costo-efectividad y que no requiere de elevada tecnología para su implementación.

\section{Declaración de conflicto de interés}

Los autores declaran no tener ningún conflicto de interés.

\section{Referencias}

Alarcón, A. \& Capafons, A. (2006). El modelo de valencia de hipnosis despierta: ¿técnicas nuevas o técnicas innovadoras? Papeles del Psicólogo, 27(2), 70-78.

Alba, L., Murillo, R., Becerra, N., Páez, N., Cañas, A., Mosquera, C., Castillo, J. S., Camacho, N., Gómez, J., García-Herreros, P. \& Bernal, L. G. (2013). Recomendaciones para la cesación de la adicción al tabaco en Colombia. Biomédica, 33, 186-204. DOI: http://dx.doi.org/10.7705/ biomedica.v33i2.651
American Asociation of Psychology (APA) Division 30, Society of Psychological Hypnosis (2014). Definition and description of hypnosis. Washington: APA. Recuperado de http://www.apadivisions.org/division-30/about/index.aspx

Asociación para el Avance de la Hipnosis Experimental y Aplicada (AAHEA) (2017). Hipnosis: una definición intencional tentativa. Valencia: AAHEA. Recuperado de http://www.aahea.net/ definicion-de-hipnosis-aahea-2017/

Ayala, C. (2010). La hipnosis como intervención para la cesación del consumo de tabaco. Revista Colombiana de Psiquiatría, 39, 224S-234S. DOI: https://doi.org/10.1016/S0034-7450(14)60278-X

Beltrán, O. A. (2005). Revisiones sistemáticas de la literatura. Revista Colombiana de Gastroenterología, 20(1), 60-69.

Bragado, C. (2009). Funcionamiento psicosocial e intervenciones psicológicas en niños con cáncer. Psicooncología, 6(2-3), 327-340.

British Society of Psychology (2001). The nature of hypnosis: a report prepared by a working party at the request of the professional affairs board of the British Psychological Society. Leicester: The British Psychological Society.

Capafons, A. (1998). Hipnosis clínica: una visión cognitivo-comportamental. Papeles del Psicólogo, (69), 1.

Capafons, A. (1999). La hipnosis despierta setenta y cuatro años después. Anales de Psicología, 15(1), 77-88

Capafons, A. (2004). Clinical applications of 'waking' hypnosis from a cognitive-behavioural perspective: from efficacy to efficiency. Contemporary Hypnosis, 21(4), 187-201. DOI: 10.1002/ch.306 Capafons, A. (2005). Hipnosis. Papeles del Psicólogo, 25(89), 1-2.

Castillo, J., Toirac, Y., Castillo, A. \& Columbié, Y. (2010). Hipnoinmunoterapia en una adolescente. Medisan, 14(8), 2048-2051.

Claros, P. (2008). Aplicación de la hipnosis en un caso de hipomotilidad colónica. Ajayu, 6(2), 165-180. 
Cos, O., Semanat, E. \& Moya, N. (2000). Sugestión y cefalea. Revista Cubana de Enfermería, 16(3), 161-166.

Gravitz, M. (1988). Early uses of hypnosis as surgical anesthesia. American Journal of Clinical Hypnosis, 30(3), 201-208. DOI: 10.1080/00029157.1988.10402733

Häuser, W., Hagl, M., Schmierer, A. \& Hansen, E. (2016). The efficacy, safety and applications of medical hypnosis. Deutsches Ärzteblatt International, 113(17), 289-96. DOI: 10.3238/ arztebl.2016.0289

Infante, M. \& Rodríguez, R. (2006). Cirugía ambulatoria en el Servicio de Cirugía General Oncológica. Revista Cubana de Cirugía, 45(3-4), 1-4.

Jensen, M. P. \& Patterson, D. R. (2014). Hypnotic approaches for chronic pain management: clinical implications of recent research findings. The American Psychologist, 69(2), 167-77. DOI: 10.1037/a0035644

Landolt, A. S. \& Milling, L. S. (2011). The efficacy of hypnosis as an intervention for labor and delivery pain: a comprehensive methodological review. Clinical Psychology Review, 31(6), 1022-1031. DOI: 10.1016/j.cpr.2011.06.002

Lanfranco, R. (2010). Hipnosis clínica y psicoterapia: una propuesta integrativa para el tratamiento de la depresión unipolar. Trastornos del Ánimo, 6(1), 37-48.

Lanfranco, R. C., Canales-Johnson, A. \& Huepe, D. (2014). Hypnoanalgesia and the study of pain experience: from Cajal to modern neuroscience. Frontiers in Psychology, 5(1126), 1-7. DOI: 10.3389/fpsyg.2014.01126

Lynn, S. J. \& Kirsch, I. (2005). Teorías de hipnosis. Papeles del Psicólogo, 25(89), 9-15.

Mac Hovec, F. J. (1975). Hypnosis before Mesmer. American Journal of Clinical Hypnosis, 17(4), 215-220. Dor: http://dx.doi.org/10.1080/00029 157.1975.10403747

Martínez-Tendero, J., Capafons, A., Weber, V. \& Cardeña, E. (2001). Rapid self-hypnosis: a new self-hypnosis method and its comparison with the hypnotic induction profile (HIP). American Journal of Clinical Hypnosis, 44(1), 3-11. DOI: http://dx.doi.org/10.1080/00029157.2001.104 03451

Mendoza, M. E. \& Capafons, A. (2009). Eficacia de la hipnosis clínica: resumen de su evidencia empírica. Papeles del Psicólogo, 30(2), 98-116.

Molina, P. J. A., Mendoza, M. E., Capafons, A. \& Llanes, B. Y. (2016). Hipnosis clínica: evaluación de su eficacia y calidad de la investigación en psicología. Revista del Hospital Psiquiátrico de La Habana, 13(2), 1-13.

Molina, P. J. A., Mendoza, M. E., Díez, G. L., Llanes, B. Y. \& Capafons, A. (2016). Hipnosis clínica: evaluación de su eficacia y calidad de la investigación en medicina. Revista del Hospital Psiquiátrico de La Habana, 13(3), 1-15. Muñiz, E., Soto, A., López, A., Orellana, M. \& Vinent, L. (2013). Hipnosis como complemento en la cirugía oral del adulto mayor. Revista Cubana de Estomatología, 49(1), 41-52.

Oliveros, S., Ortiz, C., Pérez, M., Castellanos, B. \& Cobián, A. (2015). Terapéutica holística en una paciente con gingivitis descamativa crónica. Medisan, 19(3), 428-432.

Palsson, O. S. (2015). Hypnosis treatment of gastrointestinal disorders: a comprehensive review of the empirical evidence. American Journal of Clinical Hypnosis, 58(2),134-58. DOI: 10.1080/00029157.2015.1039114

Pérez, M., Sosa, M., García, J., Pinto, M. \& Arias, D. (2008). Eficacia de la hipnoterapia contra la enuresis nocturna en niños y adolescentes. Medisan, 12(4), 1-8.

Pintar, J. \& Lynn, S. J. (2009). Hypnosis; a brief history. Nueva York: John Willey \& Sons.

Pinto, B. \& Montoya, T. (2010). Hipnosis para el control del dolor en pacientes con quemaduras. Ajayu, 8(2), 122-156.

Quiroz, J. \& Melgar, R.A. (2012). Manejo de conducta no convencional en niños: hipnosis, musicoterapia, distracción audiovisual y aromaterapia: 
revisión sistemática. Revista de Estomatologia Herediana, 22(2), 129-136.

Rodríguez, P. \& Rodríguez M. (2011). Modelo de hipnosis muy profunda colectiva y simultánea para el estudio de funciones cognitivas. Medisan, 15(6), 726-735.

Rodríguez, P. \& Rodríguez, M. (2011). Argumentación de la teoría científica: modo de funcionamiento subconsciente del cerebro en estado hipnótico. Medisan, 15(7), 1.

Samano, J. A. \& Abia, J. (1996). El recurso de la hipnosis ericksoniana en anestesia. Revista Mexicana de Anestesia, 19, 37-44.

Talley, N. J., Stanghellini, V., Heading, R. C., Koch, K. L., Malagelada, J. R. \& Tytgat, G. N. (2000). Functional gastroduodenal disorders. En D. A. Drossman, E. Corazziari, N. J. Talley, W. Grant Thompson \& W. E. Whitehead (eds.), Rome II: the functional gastrointestinal disorders (pp. 299-350) (2 ${ }^{\mathrm{a}}$ ed.). Mclean: Degnon Associates.

Tobón, S., Vinaccia \& Sandín (2003). Tratamiento psicológico de la dispepsia funcional: un análisis crítico. Terapia Psicológica, 22(2), 173-182.
Tortosa, F., Gonzales, H. \& Miguel, J. (1999). La hipnosis una controversia interminable. Anales de Psicología, 15(1), 3-25.

Uribe-Holguin, A. (2010). Psicoterapia para el manejo de eyaculación precoz no orgánica. Revista de Investigaciones en Seguridad Social y Salud, $12,85-94$

Urrutia G. (2010). Declaración Prisma: una propuesta para mejorar la publicación de revisiones sistemáticas y meta-análisis. Medicina Clínica, 135(11), 507-511.

Vallejo, M. A. (2008). Perspectiva en el estudio y tratamiento psicológico del dolor crónico. Clínica y Salud, 19(3), 417-430.

Vanhaudenhuyse, A., Laureys, S. \& Faymonville, M. E. (2014). Neurophysiology of hypnosis. Neurophysiologique Clinique, 44(4), 343-353. DOI: 10.1016/j.neucli.2013.09.006

Zayas, M., Romero, M., Alfonso T. \& Abreu, C. (2003). Hipnosis. Una modalidad terapéutica en la hipertensión arterial esencial. Archivo Médico de Camagüey, 7(2), 1-8.
Recibido: marzo 22, 2017

Aprobado: julio 13, 2017 
